\title{
Three Principles and Application of Chinese Font Design for Mobile Device Interface
}

\author{
Feng $\operatorname{Lin}^{1 *}$, Wenli $\mathrm{Lu}^{1}$ and Sanxia Wei ${ }^{1}$ \\ ${ }^{1}$ College of Fashion and Design, Donghua University, Shanghai, 200051, China
}

\begin{abstract}
Font design for interface has gradually attracted attention with the widespread use of mobile clients, especially Chinese font design is not a bold font, and the font style becomes more and more diverse. This paper takes three principles of contemporary font design as the core, and analyzes the role of emotion, structure and artistry in font design. Meanwhile, the paper also designs "Bing Tahoma" based on the three principles of font design.
\end{abstract}

\section{Introduction}

Font display in high resolution has become more and more detailed because the limitations of screen display on fonts have decreased with the advanced technology. Many reading apps gradually use serif. The era of mobile phone interface with Bold as the only font fades. However, users are not satisfied with Bold so that the demand for new fonts is increasingly urgent. Font designers need to break through the previous changes to provide mobile fonts with a more humane spirit and fonts that can meet the emotional needs of users. The new mobile font era requires designers to pay more attention to emotion, structure, and artistry.

\section{Font Design for Mobile device Interface}

Chinese characters have been sundry since ancient times. Nowadays, as mobile devices gradually become popular in people's daily lives, the mobile interface as a new medium for text communication is driving another revolution in text. The emergence of this new concept text medium brings new changes in font design.

Due to the particularity of the mobile interface display, the font type dominated by "Bold" carries most of the interfaces on the market. Chinese fonts suitable for reading and disseminating information are far less abundant than printed fonts with new media. A single typeface can't satisfy users' aesthetic diversification. Therefore, taking three principles of font design in the new era as the core, designing a new font "Bing Tahoma" can meet the user's emotional, structural and artistic needs for fonts.

\section{Three Principles of Chinese Font Design}

Users are the objects of design services [1]. Based on mobile device display, font design is a visual service for all users, and its font design needs to follow certain design principles.

\subsection{Emotion}

Based on the long-term aesthetic fatigue of a single font, users' increasing demands for the diversification of fonts in mobile interfaces are increasingly important for emotional design of fonts. The emotional design of interface fonts on mobile devices can improve the user's comfort and meet the user's visual psychological needs. In 2015, Founder's black font was launched based on the user's visual comfort. The design of the font is based on the tightening of the font, reducing the area of the text and simplifying the stroke design. In this way, the font balances the original strong industrial sense of boldface, strengthens the outline characteristics of individual fonts and the morphological differences between the text, and the reduction of the original font also increases the kerning between the new text, bringing more A good visual experience promotes the user's comfort in reading and further highlights the emotional characteristics of font design.

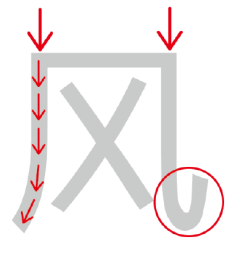

微软雅黑

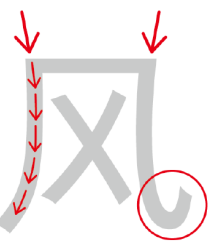

方正悠黑

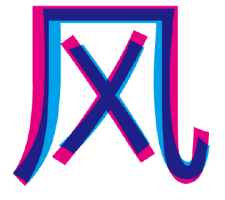

口微软雅黑 口方正悠黑
Figure 1. Different types of Chinese character "Feng" 


\subsection{Structure}

The structure is the backbone of the text [2]. Font structure is one of the important principles of interface font design. Although there are many kinds of "Bold" in the font library, they all have the same original font, which can't change the overly neat and single features. Designers need to fundamentally change the font of existing interface fonts. It is important to change its structure.

"Bing Tahoma" is a special font based on the reproduction of writing gestures, which forms a unique charm. It combines traditional printed "Tahoma" with modern black type design, and has a greater distance from the commonly seen fonts. Incorporate prettier writing in the setting of strokes, bolder breakthrough in Founder Youhei's improvement plan for zigzag strokes, drawing on the treatment of skimming, smashing, hooking and lifting from the printed "Tahoma", and weakening it After condensing into the design, not only let the font have the momentum of writing and become smart, but also make its strokes have a thick and blunt structural change. The structure of the font design can enrich the user's visual aesthetics to a certain extent.

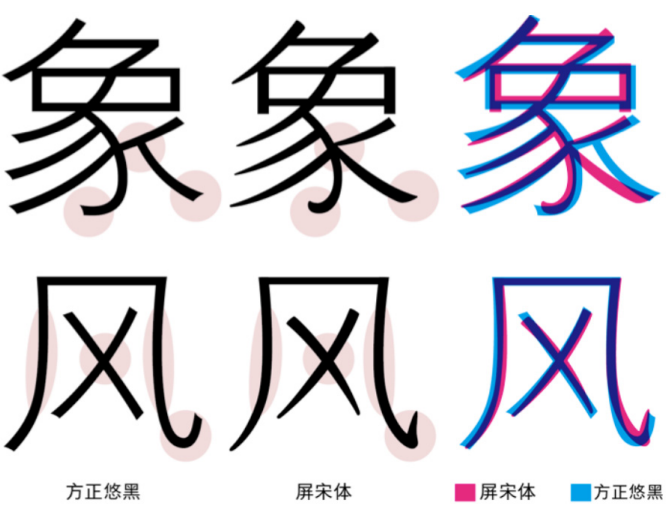

Figure 2. Comparison of "Founder Youhei" and "Bing Tahoma"

\subsection{Artistry}

The structure is one of the three elements of calligraphy, and it is also the structure that supports the writing [3]. Artistic formation can enrich the layering of the text to a certain extent. Combined with the smart brushwork of Chinese calligraphy and rigorous combination, it can show the soul of the font to a certain extent. Modern text has a set of nearly standardized patterns on the problem of font structure, and strives for the stability of the text structure, and this kind of smooth and stable structure setting may not have a good effect [4]. In the process of font art, it is based on the visual balance of the combination of text and incorporates a lively stroke, which can break through the neutral design of conventional font design.

From the writing era to the printing era to the digital era, after undergoing printing transformation and digital processing, strokes are still the most basic unit composition of a single Chinese character. When designing the text, you should not deviate from the basis of the word formation ideology of the text itself The basic form of text strokes should be retained on the basis of not deviating from the morphology of the word. Too industrial and stylized processing will destroy the ideology of the text itself.
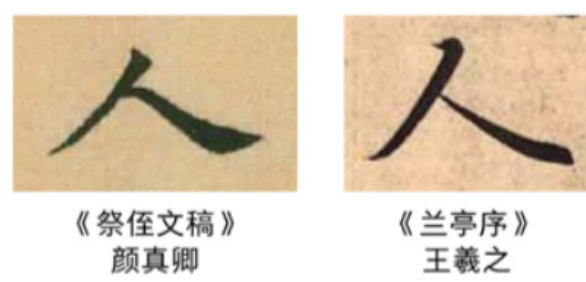

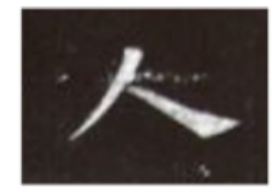

《九成宫醴泉铭碑》 欧阳询

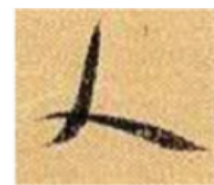

《蜀素帖》 米蒂

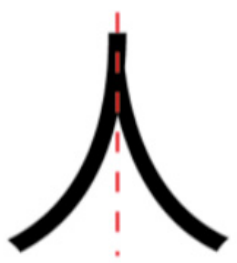

思源黑体

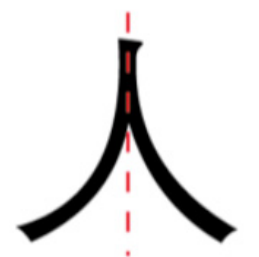

方正中等线体

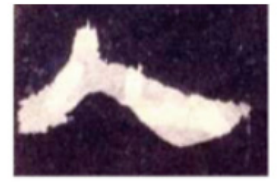

《张迁碑》

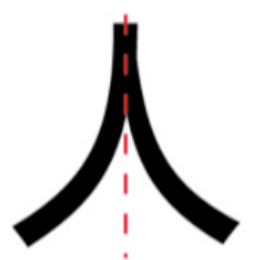

方正兰亭准黑

Figure 3. Chinese character "Ren" by Ancient Chinese calligraphers 
At present, most of the boldface fonts have been processed with different degrees of geometric strokes to meet the needs of screen display. Although most of the processing can optimize the shape of the text, it cannot be denied that The treatment of some pen shapes is debatable.

The process of pursuing the form of Chinese interface font design is actually the process of digging and expanding the special connotation of the text, changing the font of the text from reading pattern to reading text, which requires us to pay more attention to the font detail design. The most intuitive representative is our country's calligraphy fonts. The upswing, twisting, straightness, and sinking of their strokes are all different psychological activities. Taking the word "Mao" as an example, the calligrapher's upward processing of horizontal strokes is not only due to the habit of handwriting fonts, but also the result of subjective processing. Different forms of upward constitute different rhythms and rhythms of the "Mao".

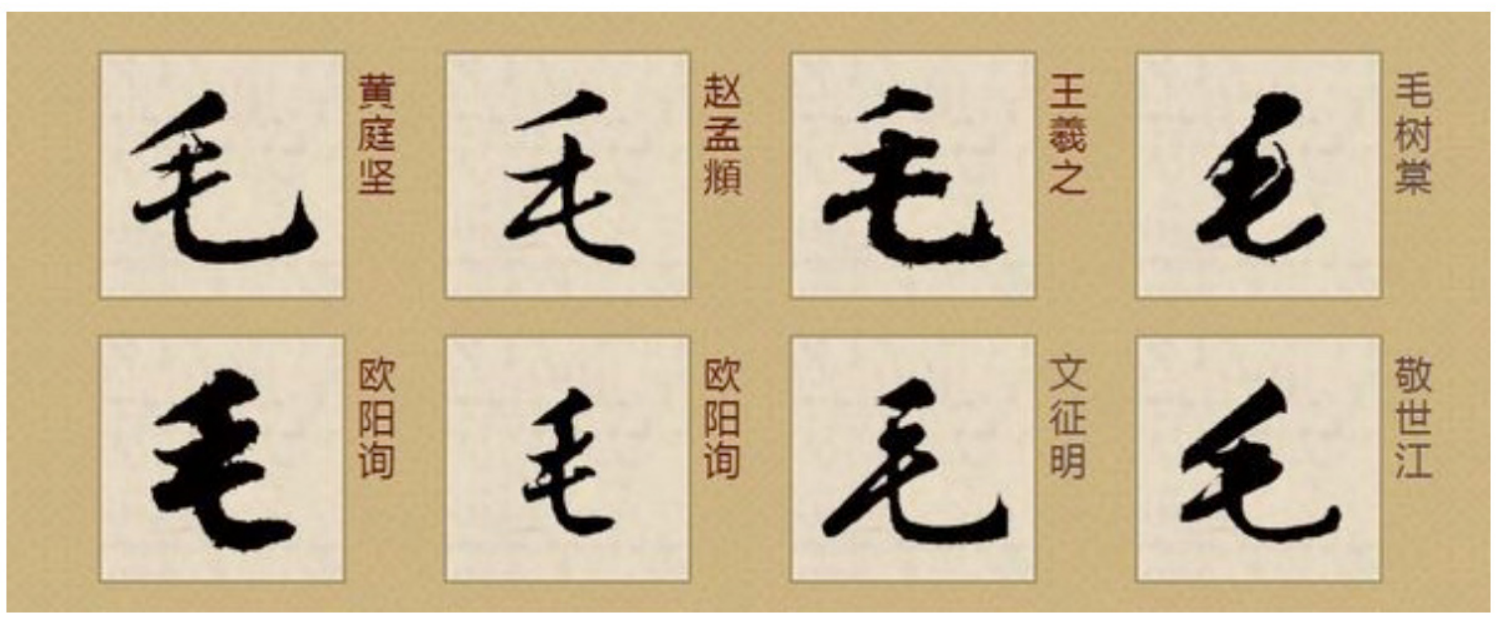

Figure 4. Chinese character "Mao" by Ancient Chinese calligraphers

\section{The Application of Chinese Font Design for Mobile device Interface}

Based on the three principles of font design, take "Bi" as an example. As can be seen from the set of "Bi" characters listed, as shown in the figure, in the process of digitizing the "Bi" characters in the existing three interface boldfaces, the "hair" horizontal drawing of the lower half of the text is simplified to Almost straight horizontal line. To a certain extent, this design breaks the original rhythm

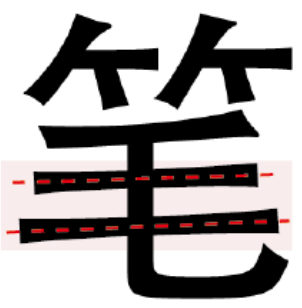

华文黑体

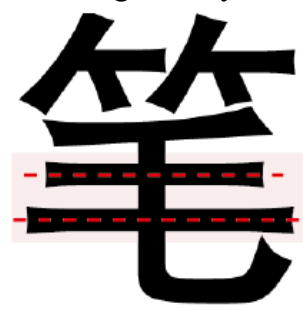

汉仪中黑简

of the "Bi" character, and it is not in line with people's habits when writing. However, after the "portrait" treatment of the Ping Song style horizontal painting, the habit of "pick up" when writing is restored, and the horizontal painting has different tilt angles according to different positions, and the degree gradually decreases from top to bottom. Infiltrate the subtle differences into the font, so that the font has a new aesthetic interest. Compared with other fonts, not only the recognition is improved, but also the rhythm is added between the strokes

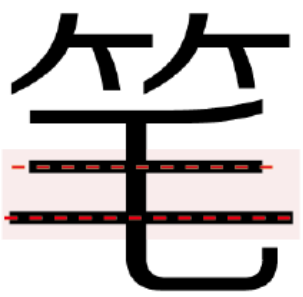

方正正纤黑

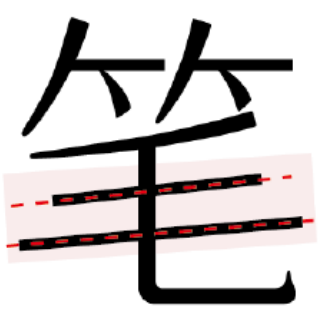

屏宋体

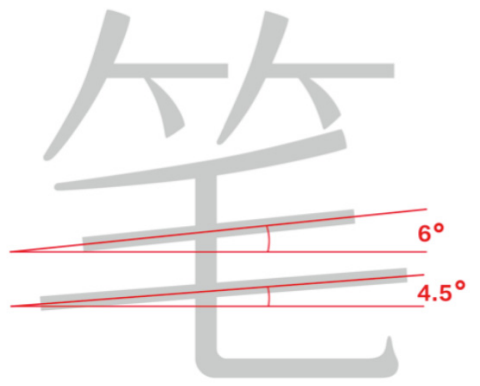

Figure 5. New Tahoma, Bing Tahoma of Chinese character "Bi" 


\section{Conclusion}

The font design in the era of digital media is easy to recognize from the initial text to the current font needs to meet the emotional needs of users. From the exploration of the design of Chinese characters by Founder Youhei, it can be seen that the design of Chinese fonts on the interface of mobile devices has certain design principles. Emotional, structured, and artistic of the font is the core idea of contemporary interface font design. In addition to meeting the easy-to-read function of text information, font designers must also focus on the spiritual needs of users. Therefore, the font of the interface of electronic devices is more soulful in the new era.

\section{Acknowledgments}

The authors would like to express their gratitude to the team of Prof. Lin for support. This work was supported by Donghua University Humanities Pre-research Project "Research on the Current Situation and Development Direction of Chinese Character Art Creation in the Context of Globalization" (107-10-0108037).

\section{References}

1. Karen C (2006). Designing Type. Yale University Press. New Haven.

2. Lawrence W (2006). Character Design for Mobile Devices. Focal Press. Oxford.

3. Chiang Y (1979). Chinese Calligraphy. Harvard University Press. Cambridge.

4. Fan H (2014). Principles of Font Design. Beijing Institute of Technology Press. Beijing. 